\title{
DERECHOS HUMANOS, EDUCACIÓN Y APRENDIZAJE ESTRATÉGICO
}

\section{HUMAN RIGHTS, EDUCATION AND STRATEGIC LEARNING}

\section{Referencia del artículo}

Aldana, K. (2020). Derechos humanos, educación y aprendizaje estratégico. Revista Científica del SEP. 3(1), 45-50. DOI: https://doi.org/10.36958/sep.v3i01.34

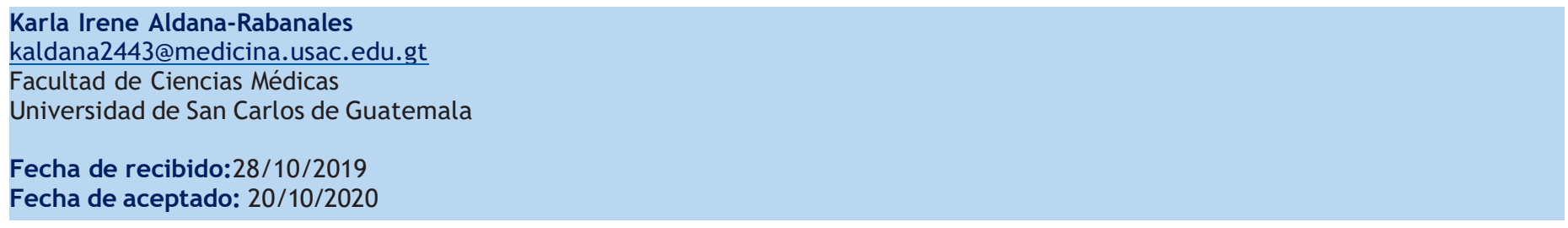

\section{RESUMEN}

El presente ensayo aborda la educación como Derecho Humano fundamentado en la dignidad de la persona. Cada persona, está en constante proceso de aprendizaje, un aprendizaje que debe ser idealmente estratégico y autónomo, para lograr el desarrollo integral que un ser humano es capaz de alcanzar y dar así- cumplimiento a los Derechos Humanos.

Para comprender el aprendizaje estratégico, es imperativo describir las estrategias de aprendizaje y centrarse en procesos cognitivos superiores. El aprendizaje es un acto cotidiano, evidente en procesos como leer y escribir, siendo éstos, entre otros, los que nos permitirán valorar la educación como un Derecho Humano inalienable.

Se propone como conclusión que el generar un ambiente de aprendizaje estratégico y autónomo, enmarcado en los Derechos Humanos es el escenario idóneo de la educación. Conclusión que pretende abrir un espacio a la reflexión, el análisis y la exploración de los constructos aquí- abordados.

\section{PALABRAS CLAVE}

Dignidad humana, evolución, aprendizaje, estrategias de aprendizaje, autorregulación

\section{ABSTRACT}

This essay addresses education as a Human Right based on the dignity of the person. Each person is in a constant learning process, a learning that should be ideally strategic and autonomous, to achieve the integral development that a human being is capable of achieving and thus comply with Human Rights.

To understand strategic learning, it is imperative to describe learning strategies and focus on higher cognitive processes. Learning is a daily act, evident in processes such as reading and writing, these being, among others, those that will allow us to value education as an inalienable Human Right.

It is proposed as a conclusion that generating a strategic and autonomous learning environment, framed in Human Rights, is the ideal setting for education. Conclusion that aims to open a space for reflection, analysis and exploration of the constructs discussed here.

\section{KEYWORDS}

Human dignity, evolution, learning, learnig strategies, selfregulation 


\section{INTRODUCCIÓN}

Hablar de educación es hablar al unísono de Derechos Humanos, es hacer valer el medio propicio para su conocimiento, apropiación y cumplimiento. En las siguientes líneas se hace un breve recorrido del surgimiento de los Derechos Humanos, centrándose en el Derecho a la Educación, como Derecho Humano de segunda generación.

Para ello, se parte de la dignidad humana y se describen los Objetivos de Desarrollo Sostenible. Existe una estrecha vinculación entre estos y el aprendizaje estratégico, que de lograrse, permite la autonomía de la persona y con ello la observancia de un ethos encaminado a la autorrealización.

\section{DESARROLLO}

Los Derechos Humanos tienen una larga historia y surgen de la necesidad del hombre de: ser, sentirse y vivir en iguales circunstancias. De hacer valer su condición única de humano, revestido de dignidad, de encontrar un marco de referencia amplio y válido para las naciones del mundo.

Los Derechos Humanos son producto de "luchas de miles de personas, de pueblos y naciones enteras; gracias a ellos, ahora podemos abrir una Constitución y encontrar una efectiva protección de tales derechos a nivel nacional, y una protección mediante convenciones Internacionales" (Sagastume, 2012, p.7).

La evolución de los Derechos Humanos está en la historia de cada pueblo, sus valores, costumbres y sistemas jurídicos. Entre los principales documentos que anteceden a los Derechos Humanos se encuentran: La Carta Magna promulgada en Inglaterra en 1215; Declaración de los Derechos de Virginia, Estados Unidos, en 1776; Declaración de los Derechos del Hombre y del Ciudadano, Francia 1789, hasta llegar al 10 de diciembre de 1948, cuando fue aprobada por consenso en la Asamblea General de la Organización de Naciones Unidas (ONU), la Declaración Universal de Derechos Humanos (Sagastume, 2012).

La Declaración Universal de Derechos Humanos (1948) enuncia en un documento, treinta artículos que hacen referencia y orientan el cumplimiento de los derechos del hombre y la mujer. Es un ideal común, considerando la libertad e igualdad del ser humano, así como la necesidad de proteger y hacer que los Estados firmantes, garanticen el cumplimiento de dichos derechos, cuya falta de reconocimiento ha causado ultrajes a la humanidad, como lo son las guerras mundiales o el conflicto armado interno, ocurrido lamentablemente en Guatemala y en otros países del mundo.
Los Derechos Humanos, se fundamentan en el reconocimiento de la dignidad de la persona, de su valor intrínseco, de saberle única e irrepetible.

La dignidad humana "es un concepto fundacional, por lo que resulta inadecuado tanto teórica como normativamente reducirla a características funcionales de la actividad de la persona, a su capacidad de tomar decisiones o al hecho de tener en cuenta su autonomía" (Organización de Naciones Unidas para la Educación, la Ciencia y la Cultura [UNESCO], 2008, p. 23).

Pelé (2015) citando a Habermas señala que: "la concepción universal e individualista de la dignidad humana que se encuentra en Kant, forma el contenido moral del cual derivaría los Derechos Humanos" (p.16). Concepción que se enlaza estrechamente al aprendizaje estratégico y autónomo. En tanto que Nussbaum, siempre en Pelé (2015), explica el sentido del respeto a la dignidad humana desde el mismo pensamiento kantiano "según el cual lo que implica el respeto de la dignidad humana es tratar al ser humano como un fin, y no como un simple medio para lograr otros fines personales" (p.17).

Es por ello, que la dignidad humana como fin y origen de los Derechos Humanos, ha de ser el marco de referencia de la educación. En el Artículo 26, la Declaración Universal de Derechos Humanos expresa el Derecho a la Educación, a la que toda persona tiene derecho y debe ser, al menos en la instrucción elemental, gratuita y obligatoria (Organización de Naciones Unidas [ONU], 1948, p. 8).

El mismo artículo se refiere a que la educación "tendrá por objeto el pleno desarrollo de la personalidad humana y el fortalecimiento del respeto a los Derechos Humanos y a las libertades fundamentales". Personalidad que ha de formarse, proporcionando el acompañamiento adecuado desde el hogar, en la escuela, sin que la educación superior sea una excepción, tomando en cuenta que el ser humano posee aspectos: "biológicos, psicológicos, sociales y aún espirituales" (Lermanda, 2007, p. 131).

UNESCO (2005) al concebir la educación como Derecho Humano, considera que la misma debe cumplir los requisitos de: Disponibilidad: siendo gratuita, obligatoria para todas las personas y que el Estado debe financiarla adecuadamente. Accesibilidad: eliminando la discriminación en general. Aceptabilidad: refiriéndose con ello a la calidad en todo el proceso de enseñanza aprendizaje. Adaptabilidad: para cada alumno o alumna. La educación abre la puerta a conocer los otros derechos de la persona humana, debiendo ser este punto uno de sus ejes transversales. 
Para dar seguimiento, UNESCO (2016), establece los objetivos para el Desarrollo Sostenible (ODS), que representan un compromiso para afrontar una serie de inquietudes universalmente compartidas y para promover el bien común. Es el resultado de un proceso de consulta inclusivo de las Naciones Unidas y que incorpora una serie considerable de aportaciones procedentes de todos los sectores de la sociedad, agentes de la comunidad internacional y de las regiones delmundo.

El ODS No. 4, corresponde a la educación, plantea "la educación como derecho fundamental y habilitador, educación como bien público, igualdad de género, para todos" (UNESCO, 2016, p.6). Abre paso a la educación inclusiva, ocupa un lugar central dentro de la agenda 2030 y da directrices para su cumplimiento por medio de siete metas y tres medios de implementación.

Los ODS son más amplios que los Objetivos del Desarrollo del Milenio (ONU, 2000) y abordan una agenda específica y mundial para la educación. Entre los aspectos a los que hace referencia se encuentra un "renovado hincapié en el aprendizaje eficaz: el ODS 4-Educación 2030 redobla su interés en el aprendizaje eficaz y la adquisición de conocimientos, capacidades y competencias relevantes" (p.9). Se dirige a la educación primaria, secundaria y a la alfabetización de jóvenes y adultos. Pero, la educación a nivel superior, no puede ser ajena a la búsqueda de un aprendizaje eficaz, por el contrario, la meta debe ser la formación integral del futuro profesional universitario, para que logre un aprendizaje eficaz que se refleje en las competencias profesionales y la capacidad para alcanzar un aprendizaje estratégico y autónomo a lo largo de la vida.

Siguiendo con el ODS 4-Educación 2030, (UNESCO, 2016) “otro aspecto innovador es la importancia que otorga a la pertinencia del aprendizaje" (p.9). Se busca que las competencias técnicas y profesionales permitan un trabajo decente y el ejercicio de la "ciudadanía global". Un profesional universitario debe incorporarse sin dificultades contando con una lectura adecuada del contexto y habilidad para ingresar a un mundo plural e interconectado.

Pero, ¿Qué es el aprendizaje? Al hablar de aprendizaje, Assmann (2002) desarrolla cuatro lecciones de la evolución y de las ciencias de la vida para la pedagogía. Se abarcan aquí dos de ellas. En la primera, define la evolución como: "la adaptabilidad de los seres vivos mediante procesos de aprendizaje" (p.50). Lo relaciona con la teoría darwiniana, retornando al origen del aprendizaje, un origen por demás interesante que tiene sus raíces en los aspectos biológicos, evolutivos y del contexto.

En la segunda lección, hace una inferencia antropológicofilosófica de la primera: "Nuestra evolución orgánica fue una evolución cognitiva, en la que desde siempre coincidieron procesos vitales y cognitivos. Así oriundos del aprendizaje, nuestra naturaleza e historia y nuestra mayor dignidad es ser aprendientes" (Assmann, 2002, p. 50).

La educación como Derecho Humano, debe dirigirse al logro del aprendizaje y con él el desarrollo pleno de la persona. La escuela, desde la elemental hasta la educación superior, tiene por reto alcanzar que cada niño, niña, joven o adulto haga vida la capacidad de "ser aprendiente". Abordando el aprendizaje como un aspecto vital en cada una de las etapas del ciclo y despertando un interés genuino en él, que permita activar el potencial que como ser humano cada uno posee.

Muñoz (2014), señala que "aprender significa adaptarse, cooperar y transformar el entorno. Es el proceso mediante el cual los cuerpos dialogan, proponen y disponen y, por ello, constituye el principio organizador de toda sociedad y de toda bilogía” (p.2).

Al ser el aprendizaje un proceso organizador, es necesario encauzarlo, de manera que, abarque los aspectos filo y ontogenéticos, permitiendo así el desarrollo de la sociedad en la cual cada persona desempeña un rol en interdependencia con los otros.

El aprendizaje, es también un sistema organizado de doble vía, que llega a los procesos cognitivos del ser humano, en donde se incorporan y proyectan la cultura y la sociedad. La educación "potencia y dota de un sentido holístico al aprendizaje (...) y es por ello que el Derecho a la educación no sólo constituye una garantía para la humanidad, sino un fundamento para la ecología y el desarrollo" (Muñoz, p. 2).

Para lograr un desarrollo que esté en armonía con la ecología debe ser sustentable, en afinidad con el medio ambiente, los recursos naturales y orientarse al logro de un proyecto ético de vida de cada ciudadano. La educación debe cumplirse como un Derecho Humano y responder a un aprendizaje eficaz, a un aprendizaje estratégico. "El aprendizaje estratégico es un concepto claramente ligado a la autonomía, y también a la responsabilidad" (Gargallo, 2012, p. 249). El aprendizaje estratégico, permite al aprendiz, independientemente de la rama del saber en la cual se forma y se desempeña, encontrar las estrategias de aprendizaje apropiadas para aplicarlas, modificarlas y recrearlas según sea el reto que el contexto le presenta. El aprendiz estratégico, es altamente capaz de autorregularse y auto-guiar procesos cognitivos superiores que le permiten generar conocimiento, tecnología, arte y ciencia.

De lograr un aprendizaje estratégico, cada aprendiz, estará en capacidad de tomar sus propias decisiones de manera autónoma y responsable. Esta es la razón por la cual la educación es el medio que guía el alcance de dicha autonomía, que debe ir enmarcada en un ethos que permita el conocimiento y observancia de los Derechos Humanos. 
Es una realidad innegable, que los avances en la tecnología y el acceso a la información, impactan las estructuras cognitivas. Gargallo (2012), argumenta que la información es solamente la materia prima para el conocimiento. El conocimiento es más complejo y para llegar a él, uno de los principios clave de la educación es la necesidad de "aprender a aprender". Lograr un aprendizaje autorregulado o estratégico, en el que entran en juego la voluntad, valorar diversos caminos cognitivos, tomar decisiones que se ajusten al contexto, permite alcanzar un aprendizaje exitoso.

Nuñez, Solano, González-Pineda y Rosário (2006) al describir el aprendizaje autónomo y permanente colocan al estudiante como la parte activa y fundamental del proceso de aprendizaje, de cómo aprende. Con lo que se remite una vez más a la necesidad de "aprender a aprender" proceso investigado por la psicología de la educación que se recoge en el constructo: “autorregulación del aprendizaje” (p.140).

El aprendizaje autorregulado o estratégico, según Gargallo (2012), implica estrategias de aprendizaje, que integran elementos afectivos-motivacionales, cognitivos, metacognitivos y contextuales, lo cual apunta al aprendizaje eficaz que buscan los Objetivos del Desarrollo Sostenible.

Monereo en 1995, definió tentativamente las estrategias de aprendizaje como "toma de decisiones, conscientes e intencionales, dirigidas a metas de aprendizaje y ajustadas a las condiciones del contexto" (Monereo, 2007, p. 499). Hace referencia a la disyuntiva entre la necesidad de integrar el aprendizaje de las estrategias en los contenidos curriculares o bien, enseñarlas directamente.

Para responder a la misma, es pertinente evaluar los avances de la ciencia, la tecnología y las diferencias generacionales, ya que como señala Chirinos (2009), es posible encontrar hasta cuatro generaciones conviviendo en el mundo laboral y cada una responde, a expectativas y actitudes que varían una de otra.

La educación debe reconocer, dentro de sus múltiples dimensiones, las necesidades laborales para las cuáles está formando a las nuevas generaciones. Promover el aprendizaje estratégico o autorregulado, sin dejar de lado los valores, la ética, aspectos socio-afectivos y contextuales, para que cumpla su misión de ser accesible, pertinente, pero sobre todo, que no pierda de vista su fin último: el pleno desarrollo de la personalidad, el conocimiento y el respeto a los Derechos Humanos.

Es necesario que la educación, tome en cuenta los aspectos de la personalidad (biológicos, psicológicos, sociales) ya que cada aprendiz difiere en el proceso de aprendizaje, activando ante una tarea concreta, procesos cognitivos y motivacionales diferentes. (García, 2012).

Estos procesos deben ir dirigidos a una meta de aprendizaje a la cual se llega por medio de las estrategias de aprendizaje: que "constituyen actividades conscientes e intencionales que guían las acciones a seguir" (Valle, Barca, González, \& Nuñez 1999, p. 428). En tal sentido, las estrategias de aprendizaje son una serie de pasos, son intencionales e implican procesos de toma de decisiones, es decir implican voluntad.

El aprendizaje de estrategias, que es a la vez aprendizaje estratégico, es más que ampliar los recursos de los estudiantes, es "que reconozcan su capacidad de evocar, adaptar o crear las estrategias alternativas que atiendan las condiciones de cada situación" (Huerta, 2007).

Los procesos cognitivos son una parte fundamental de las estrategias de aprendizaje, que se explican desde las neurociencias al incluir la motivación, análisis, planeación. (Martínez, Delgado, Guerrero, \& Hernández, 2017).

El cerebro como máquina perfecta, alberga las capacidades humanas y es en él donde tienen lugar, en condiciones ideales, el almacenamiento de la información, de los recuerdos episódicos y la elaboración de conexiones neuronales que permiten el aprendizaje. Radica aquí la importancia del proceso educativo desde antes del nacimiento, hasta las etapas más avanzadas del ciclo vital, en un aprendizaje permanente.

Un ejemplo de cómo el aprendizaje estratégico está vinculado a los Derechos Humanos y una de las formas de cumplir con el Derecho de la educación, es la enseñanza y el aprendizaje armónico de la lectura y escritura. Para lograrlo, es necesario un aprestamiento adecuado tomando en cuenta que este es el cimiento de los aprendizajes posteriores y la puerta al mundo del conocimiento, la cultura, el arte, la ciencia, los valores y los derechos. Villalobos (2007) presentó una ponencia sugestiva, en la cual describe el proceso de aprendizaje de la escritura en toda su complejidad, y como en ella se reflejan estrategias de aprendizaje y procesos cognitivos superiores, evidenciando la necesidad de que la misma se promueva infatigablemente.

El aprendizaje estratégico, autónomo o de estrategias de aprendizaje es el recurso para el conocimiento y reflexión de los Derechos Humanos. Pero es preciso, como menciona Pérez (2013) “promover y transmitir conocimientos, actitudes y acciones de y para los Derechos Humanos" (p.84). Es decir, conocerlos y practicarlos en la simpleza de la cotidianeidad y en lo complejo del tejido social, siendo aquí donde se educa, aprende y se crece como persona individual y autónoma. 


\section{CONCLUSIÓN}

Generar un ambiente de aprendizaje estratégico y autónomo, enmarcado en los Derechos Humanos es el escenario idóneo de la educación.

Derecho que debe ser un reto para el sistema educativo, teniendo presente que su fin es el desarrollo pleno de la personalidad, en sus esferas: biológica, psicológica y social. Promoviendo así, un ethos que permita la convivencia pacífica que parte del respeto a la dignidad humana.

\section{REFERENCIAS BIBLIOGRÁFICAS}

Assmann, H. (2002). Placer y ternura en la educación. Madrid, España: Narcea.

Chirinos, N. (2009). Características generacionales y losvalores. Su impacto en lo laboral. Observatorio laboral. Revista venezonala, 133-153. Recuperado de http: //www.redalyc.org/pdf/2190/Resumenes/Resumen _219016846007_1.pdf

García, M. (2012). La autorregulación académica como variable explicativa de los procesos de aprendizaje universitario. Profesorado. Revista de currículum y formación del profesorado, 16(1), 203-221. Recuperado de http: / / www.redalyc.org/articulo.oa?id=56724377012

Gargallo, B. (2012). Un aprendiz estratégico para una nueva sociedad. Teoría de la educación, educación y cultura en la sociedad de la información, 13(2), 246272. https://doi. org/10.4272/84-9745-093-0.ch18

Huerta, M. (2007). Aprendizaje estratégico, una necesidad del siglo XXI. Revista iberoamericana de educación, 42(1), 1-13. Recuperado de https://rieoei.org/historico/ deloslectores/1541Huerta.pdf

Lermanda, C. (2007). Aprendizaje basado en problemas (ABP): Una experiencia pedagógica en medicina. REXE. Revista de estudios y experiencias en educación. (11), 127-143. Recuperado de http://www.rexe.cl/ojournal/index.php/ rexe/article/view/197/204

Martínez, F., Delgado, U., Guerrero, G., \& Hernández, E. (2017). Estrategias de aprendizaje, de la educación a la neurocognición. ConCiencia, 2, 58-66. Recuperado de https: / /www.researchgate.

net/publication/328361261_Estrategias_de_aprendizaje_ de_la_educacion_a_la_neurocognicion

Monereo, C. (2007). Hacia un nuevo paradigma del aprendizaje estratégico: el papel de la mediación social, del self y de las emociones, 497-534. https://doi.org/10.25115/ejrep.v5i13.1250.
Muñoz, V. (2014). El derecho humano a la educación. Sinéctica. Revista electrónica de educación (42), 1-10. Recuperado de: http://www.redalyc.org/pdf/998/99829581003.pdf

Nuñez, J., González-Pineda, J., Rosário, P., \& Solano, P. (2006). El aprendizaje autorregulado como medio y meta de la educación. Papéles de psicólogo, 27(3), 139-146. Recuperado de: http: / /www.redalyc.org/pdf/778/77827303.pdf

Organización de Naciones Unidas (ONU). (1948). Declaración universal de derechos humanos. París: Naciones Unidas.

Organización de Naciones Unidas (ONU). (2000). Objetivos del desarrollo del milenio. Nueva York: ONU.

Organización de las Naciones Unidas para la Educación, la Ciencia y la Cultura (UNESCO). (2016). Desglosar el objetivode desarrollo sostenible 4. Educación 2030. Guía. New York: UNESCO. Recuperado de: https://unesdoc.unesco.org/ ark:/48223/pf0000246300_spa

Organización de las Naciones Unidas para la Educación, la Ciencia y la Cultura (UNESCO). (2008). Programa de base de estudios de bioética. Montevideo: Programa de Bioética y Ética de la Ciencia. Recuperado de: https://redbioetica.com.ar/wpcontent/uploads/2018/11/Bioetica_Base.pdf

Pelé, A. (2015). Kant y la dignidad humana. Revista Brasileira de Estudos Políticos (111), 15-46. https://doi.org/10.9732/P.0034-7191.2016V111P15

Pérez, T. (2013). Aproximaciones al estado de la cuestión de la investigación en educación y derechos humanos. Revista RIIEP,79-101. https://doi.org/10.15332/s1657107x.2013.0001.05 Sagastume, A. (2012). Introducción a los derechos humanos.Guatemala: Editorial Universitaria.

UNESCO Etxea. (2005). La Educación como derecho humano. (Bakun, Trad.) España, Gobierno Vasco: Graficolor.

Valle, A., Barca, A., González, R., \& Nuñez, J. (1999). Las estrategias de aprendizaje. Revisión teórica y conceptual. Revista latinoamericana de psicología., 31(3), 425-461. Recuperado de: https: / / www.redalyc.org/pdf/805/80531302.pdf

Villalobos, J. (Del 06 al 09 de junio de 2007). Identificación de estrategias de aprendizaje. Identificación de estrategias de aprendizaje, (págs. 1-14). Montevideo. Recuperado de: http://www.lecturayvida.fahce.unlp.edu.ar/numeros/a2 8n3/28_03_Villalobos.pdf 


\section{Sobre autora}

\section{Karla Irene Aldana Rabanales}

Es Licenciada en Psicología, Máster en Educación de Valores. Inició su carrera docente a nivel universitario, hace 21 años, en la Escuela de Ciencias Psicológicas, Universidad de San Carlos de Guatemala (USAC), en el área de Psicología Educativa. Actualmente profesora titular del Área de Formación y Desarrollo del Personal Académico (AFDPA), de la Escuela de Estudios de Postgrado (EPP) de la Facultad de Ciencias Médicas de la USAC. Investigación educativa, en la línea de: estrategias de aprendizaje.

Copyright (c) 2020 Karla Irene Aldana Rabanales

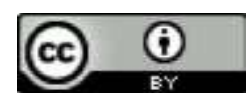

Este texto está protegido por una licencia Creative Commons 4.0.

Usted es libre para Compartir - copiar y redistribuir el material en cualquier medio o formato - y Adaptar eldocumento - remezclar, transformar y crear a partir del material- para cualquier propósito, incluso comercialmente, siempre que cumpla la condición de:

Atribución: Usted debe reconocer el crédito de una obra de manera adecuada, proporcionar un enlace a la licencia,e indicar si se han realizado cambios. Puede hacerlo en cualquier forma razonable, pero no de forma tal que sugieraque tiene el apoyo del licenciante o lo recibe por el uso que hace.

$\underline{\text { Resumen de licencia }}$ - Texto completo de la licencia 